\title{
Multimodal Imaging Findings and Treatment with Dexamethasone Implant in Three Cases of Idiopathic Macular Telangiectasia Type 1
}

\author{
Paola Cirafici $^{a}$ Maria Musolino ${ }^{a}$ Michela Saccheggiani $^{a}$ \\ Carlo Enrico Traverso ${ }^{a}$ Massimo Nicolò ${ }^{a}, b$ \\ aDi.N.O.G.Mi, Clinica Oculistica, Università di Genova, Ospedale Policlinico San Martino \\ IRCCS, Genova, Italy; ${ }^{\text {b} F o n d a z i o n e ~ p e r ~ l a ~ M a c u l a ~ O n l u s, ~ G e n o v a, ~ I t a l y ~}$
}

\section{Keywords}

Imaging $\cdot$ MacTel $1 \cdot$ Dexamethasone implant $\cdot$ Macular edema $\cdot$ Retina

\begin{abstract}
This is a report of 3 patients diagnosed with idiopathic macular telangiectasia type 1 (MacTel 1) at Medical Retina Center of the University of Eye Clinic of Genova and then prospectically followed for a mean time of 26 months between 2016 and 2019. It is the first report of patients affected by MacTel 1 treated with dexamethasone (DEX) implant as a first choice of treatment. Aim of our study is to better characterize the disease using a multimodal wide-field imaging and to determine efficacy of DEX implant on MacTel 1 in terms of central macular thickness (CMT) and best-corrected visual acuity (BCVA). MacTel 1 is a rare unilateral disease, characterized by telangiectatic retinal capillaries, cystoid macular edema, and lipid deposition occurring temporal to the fovea. Patients underwent a comprehensive ophthalmic examination, BCVA, swept-source optical coherence tomography (SS-OCT), SS-OCT angiography (SS-OCTA), ultrawidefield (UWF) color, and fluorescein angiography (FA) fundus photograph. All the patients presented monolateral reduced BCVA and macular edema with increased CMT evaluated by SS-OCT. With SS-OCTA, we showed that the telangiectasia-associated vascular changes originate in the deep retinal vascular plexus and as a consequence macular edema and exudation develop causing vision loss. Furthermore, UWF imaging helped us to highlight vascular changes typical of Coats Disease at the far retinal periphery. All the patients were treated with DEX intravitreal implant, showing a decrease in CMT and a stabilization of visual acuity. Due to the recurrent nature of macular edema, patients underwent a mean of 4 DEX implants during the follow-up period. In order to address the clinical features of this uncommon disease avoiding diagnostic errors, it might be important to use a multimodal imaging approach. The anatomical and functional beneficial effects of DEX implant were well evident although transient.
\end{abstract}


Cirafici et al.: Imaging and Treatment in MacTel 1

\section{Introduction}

Idiopathic macular telangiectasia type 1 (MacTel 1) is a rare unilateral and predominantly affecting male ocular pathology [1]. MacTel 1 is characterized by telangiectatic retinal capillaries, cystoid macular edema, and lipid deposition occurring temporal to the fovea. Macular edema and exudation are the main causes of visual loss [2]. Here, we describe multimodal imaging findings and treatment strategies employed in a case series of patients affected by MacTel 1.

\section{Case Reports}

We report 3 cases of healthy male patients prospectically followed at the Medical Retina Centre of the University of Eye Clinic of Genova. Mean age was 54 years (range 51-59 years). All the patients exhibited unilateral gradual vision loss. Patients underwent a comprehensive ophthalmic examination, best-correct visual acuity (BCVA), swept-source optical coherence tomography (SS-OCT); SS-OCT angiography (SS-OCTA); ultra-widefield (UWF) color, and fluorescein angiography (FA) fundus photograph.

Ophthalmoscopy showed small hemorrhages and microaneurysms located temporal to the fovea, as well as lipid exudates. Early phase FA revealed hyperfluorescent saccular dilatations which exhibited leakage during late phase. SS-OCT showed increased macular thickness and intraretinal cysts. SS-OCTA showed a mild reduction in the capillary network density of the superficial retinal layer, as well as multiple telangiectatic dilated vessels in the deep retinal layers [3] in which it was possible to detect blood flow (shown in Fig. 1). Dilated and dropout retinal capillaries as well as mild fibrosis in peripheral retinal areas were clearly visible through UWF fundus and FA in all 3 cases (shown in Fig. 2). No abnormalities were detected in the fellow eye of either patient.

\section{Case 1}

A 59-year-old male was the first MacTel 1 patient observed on December 30, 2016. At presentation, BCVA was 20/32 and central macular thickness (CMT) was $398.89 \mu \mathrm{m}$. Since no well-established treatment options were available [4], the patient initially underwent a 1-month observational period at the end of which no clinical changes were detected, and the affected eye was treated with DEX implant. At 1-month follow-up, SS-OCT revealed a significant regression of the exudation with a CMT of $331.89 \mu \mathrm{m}$ despite stable BCVA. Five months later, macular edema recurred (CMT $404.11 \mu \mathrm{m}$ ) and the patient underwent a second DEX implant. During follow-up, macular edema recurred twice and he was treated with a third and a fourth DEX implant. BCVA gradually decreased to 20/100 due to cataract development. At the last visit, the patient underwent phacoemulsification with a combined intravitreal DEX implant.

Case 2

A 51-year-old male presented with a BCVA of 20/32 and a CMT of $435.22 \mu \mathrm{m}$ on November 2 , 2017. He was treated with DEX implant. At 1-month follow-up, there was a significant regression of exudation (CMT $=350.44 \mu \mathrm{m}$ ), and BCVA improved to 20/25. UWF imaging 1-month following treatment showed a reduction of the fibrotic temporal areas previously detected.UWF-FA performed after DEX implant confirmed the presence of peripheral saccular dilatations and increased of the intercapillary spaces, capillary dropout and retinal nonperfusion in the peripheral temporal quadrant were also highlighted. The peripheral nonperfused areas were treated with light laser photocoagulation. Four months after the first 

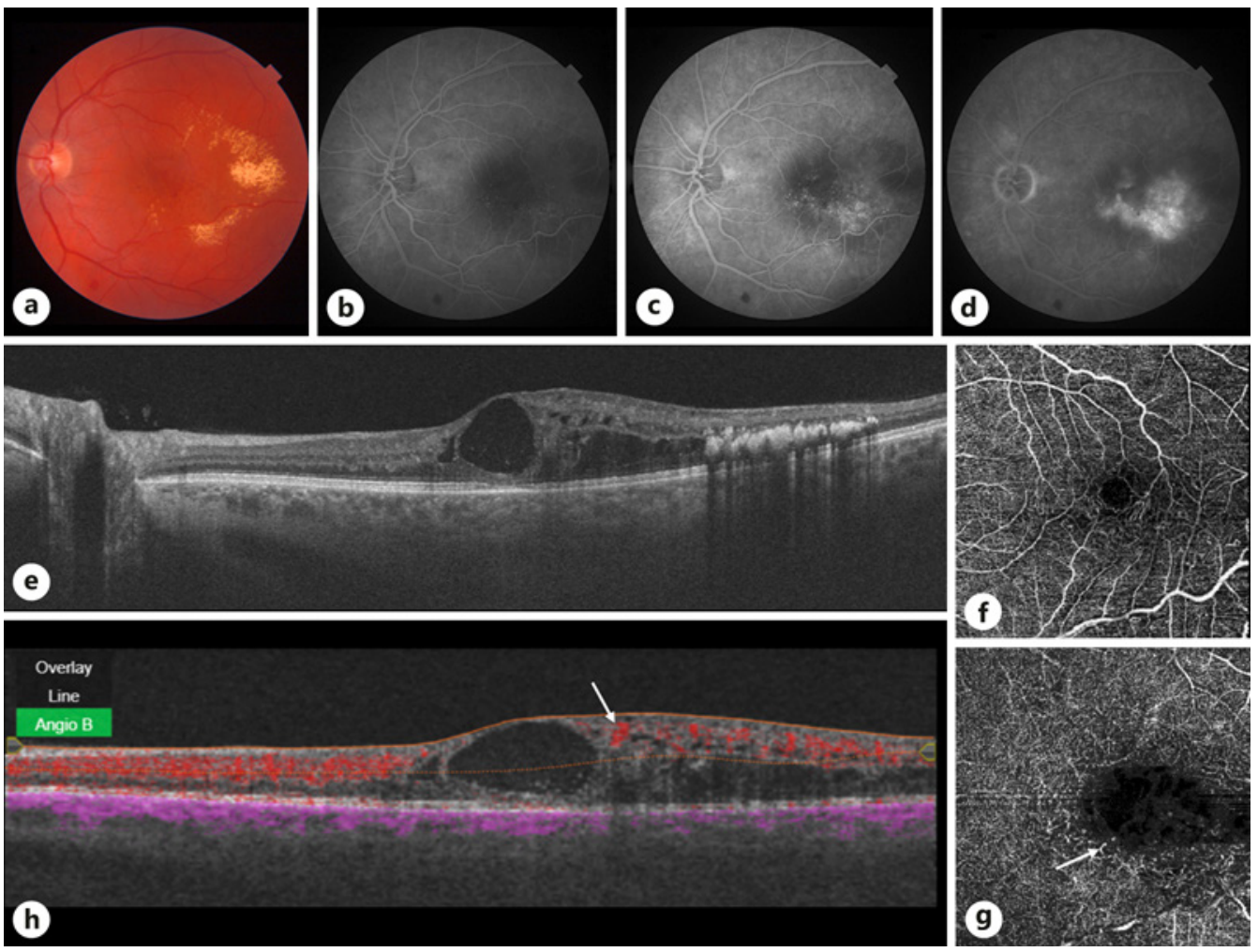

Fig. 1. Case 2 a. Fundus examinations of the affected showed small hemorrhages, microaneurysms, and lipid exudates located temporal to the fovea. b Early phase FA revealed hyperfluorescent saccular dilatations which exhibited profuse leakage during intermediate (c) and late phase (d). e Swept-source OCT showed increased central retinal thickness and intraretinal cysts. Swept-source OCT angiography showed a mild reduction in the capillary network density of the superficial retinal layer (f), as well as multiple telangiectatic dilated vessels in the deep retinal layers $(\mathbf{g})$ in which it was possible to detect blood flow at structural OCT (h, arrow). FA, fluorescein angiography.

treatment, despite stable BCVA, intraretinal edema recurred (CMT $427.56 \mu \mathrm{m}$ ) and the patient was treated with a second DEX implant. A month later, macular edema decreased (CMT $371.56 \mu \mathrm{m})$ and BCVA was stable. Five months after the second treatment, macula edema recurred (CMT $466.11 \mu \mathrm{m}$ ), BCVA decreased to 20/40, and the patient was treated with a third intravitreal DEX implant. A month later, macular edema decreased (CMT $366.78 \mu \mathrm{m}$ ) and BCVA stable. Six months after the third treatment, despite stable BCVA, macula edema recurred again (CMT $525.89 \mu \mathrm{m}$ ). The patient was treated with a fourth intravitreal DEX implant.

\section{Case 3}

A 52-year-old male was diagnosed with MacTel 1 on February 1, 2018. Baseline BCVA was $20 / 100$, and CMT was $465.89 \mu \mathrm{m}$. He was treated with DEX implant at the first observation. At 1 and 2 months after treatment, macular edema disappeared (CMT 332.33 and $328.56 \mu \mathrm{m}$, respectively) and BCVA improved to 20/32. Three months after treatment, macular edema recurred (CMT $361.67 \mu \mathrm{m}$ ) despite stable BCVA. Patient underwent a second DEX implant. Two months later, BCVA was stable and macular edema decreased (CMT 332.44 $\mu \mathrm{m})$. However, 3 months after the second DEX implant, macular edema recurred again (CMT $428.78 \mu \mathrm{m}$ ). The patient was treated with a third DEX implant, and after 1 month, macular edema decreased (CMT $331.56 \mu \mathrm{m}$ ) and BCVA remains stable. Three months after the third 


\section{Case Reports in Ophthalmology}
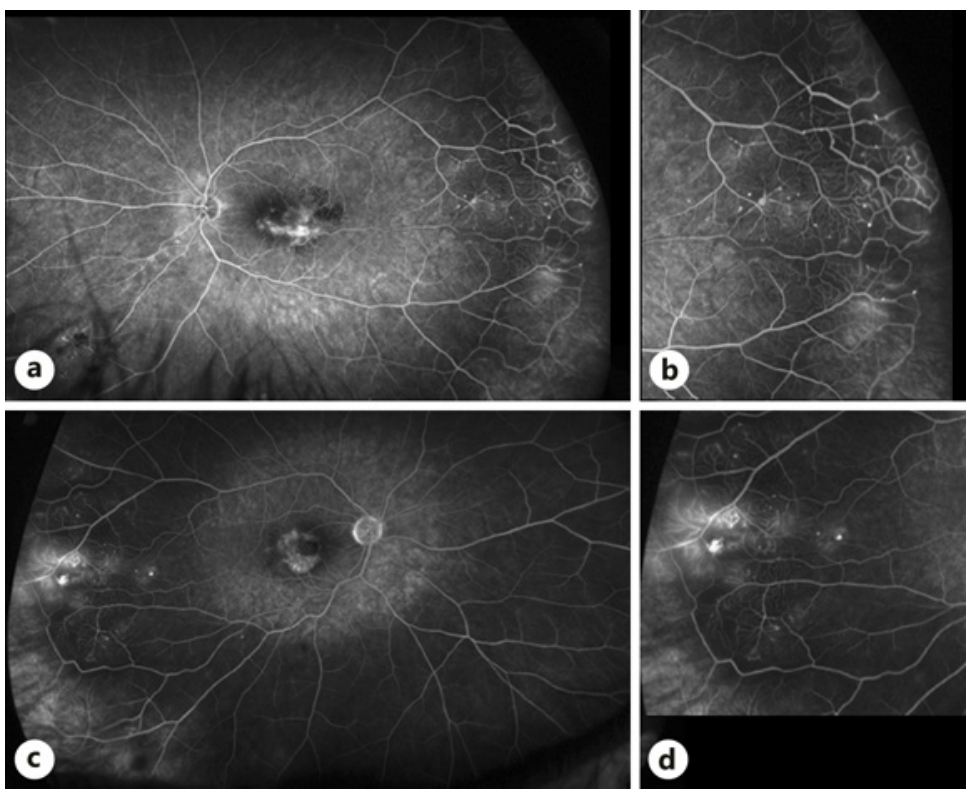

Fig. 2. Dilated and dropout retinal capillaries in peripheral retinal visible through UWF fundus FA. Case 2 (a, b); Case 3 (c, d). UWF, ultra-widefield; FA, fluorescein angiography.

treatment, macular edema (CMT $387.33 \mu \mathrm{m}$ ) recurred. A fourth DEX implant, implant was administered. At the last follow-up visit, 2 months after the fourth DEX implant, BCVA was 20/32 and macular exudation was significantly reduced (CMT $328.33 \mu \mathrm{m}$ ).

\section{Discussion}

MacTel 1 is considered a variation of Coats Disease sometimes confused with other more common macular disease [5]. In contrast to Coats disease, MacTel 1 starts with telangiectasia and saccular dilation at the temporal side of the fovea. In order to address the clinical features of this uncommon disease avoiding diagnostic errors, it might be important to use a multimodal imaging approach. We showed that the telangiectasia-associated vascular changes originate in the deep retinal vascular plexus [6] and as a consequence macular edema and exudation develop causing vision loss. Furthermore, UWF imaging helped us to highlight vascular changes typical of Coats Disease at the far retinal periphery. Those changes would not be probably imaged using traditional fundus camera, and their recognition is very important in order to address a precise diagnosis.

There are no established treatments for MacTel 1. Laser photocoagulation can be performed on accessible ischemic areas as well as to target leaky capillary dilatation. However, parafoveal retinal scarring as well as recurrence can occur [7]. Previous reports used antiVEGF drugs [8-10] with inconstant results probably because VEGF-A is not overexpressed in this disease. On the contrary, placental-growth factor was found to be increased in the humor aqueous of MacTel 1 patients and might be the reason for a better clinical response with intravitreal aflibercept [11].

In 2006, Cakir et al. [12] described the first 3 patients affected by MacTel 1 treated with a single intravitreal injection of triamcinolone acetonide showing angiographic improvement of the macular edema and minimal decrease in retinal thickness on OCT. Since then, 4 more reports [13-16] for a total of 7 cases of MacTel 1 have been treated with DEX implants all of

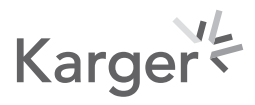




\section{Case Reports in Ophthalmology}
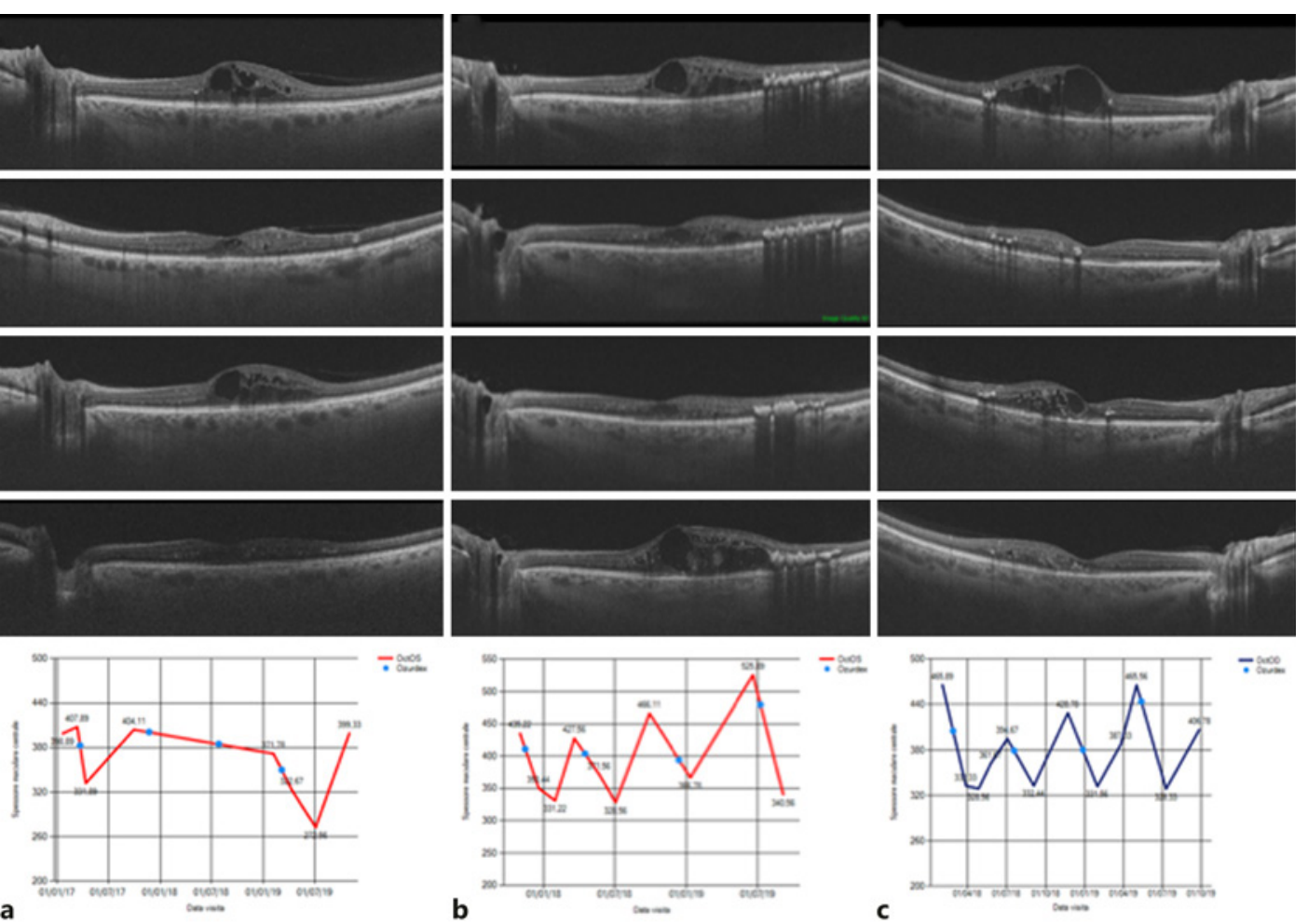

Fig. 3. Change in CMT after intravitreal injections of DEX implant in 3 cases of MacTel 1. Case 1 in column (a); Case 2 in column (b); Case 3 in column (c). CMT, central macular thickness.

them as a second-line treatment after failure of anti-VEGF treatment. Although potential adverse effects like increase in intraocular pressure and development of cataracts might be possible, they are usually well managed and overcome by the significant although transient reduction in macular edema and exudation. None of our patients had an increase in intraocular pressure although 1 developed cataract. However, the anatomical and functional beneficial effects were well evident although transient (shown in Fig. 3). Possible explanations for the effect of intravitreal long-acting steroids in inducing the decrease of macular edema and exudation might be correlated to the overexpression of inflammatory cytokines as well as it happens in diabetic or retinal vein occlusion macular edema.

In conclusion, MacTel 1 diagnosis might not be easily obtained without using new diagnostic technologies like OCT angiography or UWF imaging. Treatment is usually addressed to the resolution of macular edema and exudation, and long-acting steroids might be effectively used. More studies are needed in order to better delineate the cytokines profile and finding new therapeutic strategies.

\section{Statement of Ethics}

This study has been performed with the appropriate participants' informed consent in compliance with the Helsinki Declaration. All the patients have given their written informed consent to publish their case, including publication of images. 
Cirafici et al.: Imaging and Treatment in MacTel 1

\section{Conflict of Interest Statement}

The authors have no conflicts of interest to declare.

\section{Funding Sources}

This study did not receive any relevant funding source.

\section{Author Contributions}

Paola Cirafici conceived of the presented idea, collected and analyzed the data, drafted the article, and critically reviewed the work. Maria Musolino collected the data and helped to draft the article. Michela Saccheggiani collected the data and helped to draft the article. Massimo Nicolò conceived of the presented idea, analyzed the results, and contributed to the writing and critically reviewing of the manuscript. Carlo Enrico Traverso critically reviewed the work, supervised the project, and contributed to the design and implementation of the research. All the authors approve the final version of the manuscript for publication.

\section{References}

1 Yannuzzi LA, Bardal AM, Freund KB, Chen KJ, Eandi CM, Blodi B. Idiopathic macular telangiectasia. Retina. 2006;32 Suppl 1(4):450-60.

2 Nowilaty SR, Al-Shamsi HN, Al-Khars W. Idiopathic juxtafoveolar retinal telangiectasis: a current review. Middle East Afr J Ophthalmol. 2010;17(3):224-41.

3 Pappuru RR, Peguda HK, Dave VP. Optical coherence tomographic angiography in type 1 idiopathic macular telangiectasia. Clin Exp Optom. 2018;101(1):143-4.

4 Chatziralli IP, Sharma PK, Sivaprasad S. Treatment modalities for idiopathic macular telangiectasia: an evidence-based systematic review of the literature. Semin Ophthalmol. 2017;32(3):384-94.

5 Osaka R, Shiragami C, Ono A, Kobayashi M, Takasago Y, Yamashita A, et al. Clinical features of treated and untreated type 1 idiopathic macular telangiectasia without the occurrence of secondary choroidal neovascularization followed for 2 years in japanese patients. Retina. 2018;38 Suppl 1(Suppl 1):S114-22.

6 Mao L, Weng S, Gong Y, Yu SQ. Optical coherence tomography angiography of macular telangiectasia type 1: comparison with mild diabetic macular edema. Lasers Surg Med. 2017;49(3):225-32.

7 Stoffelns BM, Schoepfer K, Kramann C. Idiopathic macular telangiectasia: follow-up with and without laser photocoagulation. Klin Monbl Augenheilkd. 2010;227:252-6.

8 Gamulescu MA, Walter A, Sachs H, Helbig H. Bevacizumab in the treatment of idiopathic macular telangiectasia. Graefes Arch Clin Exp Ophthalmol. 2008;246(8):1189-93.

9 Koay CL, Chew FL, Visvaraja S. Bevacizumab and type 1 idiopathic macular telangiectasia. Eye. 2011;25(12): 1663-5. Author reply 1665.

10 Ciarnella A, Verrilli S, Fenicia V, Mannino C, Cutini A, Perdicchi A, et al. Intravitreal ranibizumab and laser photocoagulation in the management of idiopathic juxtafoveolar retinal telangiectasia type 1: a case report. Case Rep Ophthalmol. 2012;3(3):298-303.

11 Kowalczuk L, Matet A, Dirani A, Daruich A, Ambresin A, Mantel I, et al. Efficacy of intravitreal aflibercept in macular telangiectasia type 1 is linked to the ocular angiogenic profile. Retina. 2017;37(12):2226-37.

12 Cakir M, Kapran Z, Basar D, Utine CA, Eroglu F, Perente I. Optical coherence tomography evaluation of macular edema after intravitreal triamcinolone acetonide in patients with parafoveal telangiectasis. Eur J Ophthalmol. 2006;16(5):711-7.

13 Erdoğan G, Aydoğan T, Ünlü C, Ergin A. Dexamethasone implant for the treatment of type 1 idiopathic macular telangiectasia. J Ocul Pharmacol Ther. 2016;32(4):211-5.

14 Loutfi M, Papathomas T, Kamal A. Macular oedema related to idiopathic macular telangiectasia type 1 treated with dexamethasone intravitreal implant (ozurdex). Case Rep Ophthalmol Med. 2014;2014:231913.

15 Sandali O, Akesbi J, Rodallec T, Laroche L, Nordmann JP. Dexamethasone implant for the treatment of edema related to idiopathic macular telangiectasia. Can J Ophthalmol. 2013;48(4):e78-80.

16 Lei S, Lam WC. Efficacy and safety of dexamethasone intravitreal implant for refractory macular edema in children. Can J Ophthalmol. 2015;50(3):236-41. 